\title{
Analyzing Web pages visual scanpaths: between and within tasks variability
}

\author{
Gautier Drusch $^{a}$ and J. M. Christian Bastien ${ }^{a^{*}}$ \\ ${ }^{a}$ Département de Psychologie, Université Paul Verlaine-Metz, UFR SHA, BP 30309, Île du Saulcy, 57006 Metz, \\ France
}

\begin{abstract}
In this paper, we propose a new method for comparing scanpaths in a bottom-up approach, and a test of the scanpath theory. To do so, we conducted a laboratory experiment in which 113 participants were invited to accomplish a set of tasks on two different websites. For each site, they had to perform two tasks that had to be repeated ounce. The data were analyzed using a procedure similar to the one used by Duchowski et al. [8]. The first step was to automatically identify, then label, AOIs with the mean-shift clustering procedure [19]. Then, scanpaths were compared two by two with a modified version of the string-edit method, which take into account the order of AOIs visualizations [2]. Our results show that scanpaths variability between tasks but within participants seems to be lower than the variability within task for a given participant. In other words participants seem to be more coherent when they perform different tasks, than when they repeat the same tasks. In addition, participants view more of the same AOI when they perform a different task on the same Web page than when they repeated the same task. These results are quite different from what predicts the scanpath theory.
\end{abstract}

Keywords: eye-tracking, scanpath theory, scanpath variability, user behavior, web exploration

\section{Introduction}

Noton and Stark's [16] scanpath theory asserts that eye-movements tends to follow a "habitually preferred path" when people are exploring a visual scene. This means that people will exhibit approximately the same visualization sequence if they look at a particular stimulus twice. Josephson and Holmes [14] tested this theory on Web pages exploration. Their results where mixed. It appeared that a set of participants followed a preferred path, but sometimes, there were more within participants' variation in the scanpaths than variations between participants' paths. These results have raised important questions: how can we measure scanpaths variability and how can we compare different scanpaths. In other words, how can we show and measure similar and different scanpaths, or how can we classify people according to their scanpaths? These questions are worth answering both from a theoretical point of view and a practical point of view, i.e., for providing developers with design guidelines.
The aim of this paper is to test the scanpath theory and to propose a new method for comparing scanpaths.

\section{The scanpath}

Studies on scanpath aim at validating a model of eye movements to explain the behavior of users on the Web. Initially, these studies were conducted on relatively simple pictures. Now, some researchers are wondering if this model is still valid for complex documents such as Web pages, but also how this model can help analyze ocular behaviors.

Noton and Stark [16] were the first to introduce the notion of scanpath. It is the "habitually preferred path" for a given participant when he looks at a familiar object. So, the scanpath is a typical pattern of eye-movements when subjects recognize an object. Thus, according to these authors, each human would have a specific scanpath for any specific object.

\footnotetext{
*Corresponding author. E-mail: Christian.bastien@univ-metz.fr
} 
Their idea is that the scanpath cannot be reduced to a simple eye movement aiming at aligning areas of the visual scene on the retina, but that the scanpath is a component of the memory for pattern recognition. Indeed, after the presentation of a new visual stimulus to a subject, the sensory and memory information is encoded (encoding scanpath). In a second presentation of the stimulus (recognition phase), the subject matches its internal representation of the stimulus with the stimulus presented.

In other words, the subject will reproduce the sequence of eye movements stored in memory by checking if the characteristics of the visual scene encoded are present. Once the scanpath is reconstituted, the subject recognizes the object. Thus, the scanpath can be viewed as a form of procedural knowledge.

According to Noton and Stark [16], this conclusion is supported by two observations. On the one hand, the scanpaths were inconsistent between subjects, which suggests that they were not solely determined by the characteristics of the visual scene. On the other hand, the scanpath of a given subject differed according to the images presented, indicating that the scanpath was not stereotypical, but a consistent pattern of visualization for a particular image.

Josephson and Holmes [14] have tested the scanpath' theory on the Web. To do this, they asked subjects to view three different Web pages (respectively from a portal page, an advertising page and a news page) 48 hours apart.

To compare scanpaths within and betweensubjects, a string-edit method was used (see section 3). Results showed that scanpaths for the news Web page were more similar to each other than were the most similar sequences for the portal or advertising Web pages. According to the authors, these results suggest a lack of stability over time of the subject's scanpath and that there is a confounding memory effect. But for the other Web pages (portal and advertising), there was a strong within-subjects resemblance between scanpaths.

These findings suggest that subjects have their own viewing habits, but also that other variables seem to play a role on scanpaths such as memory or the characteristics of Web pages. However, this study had only a few participants and, according to the authors, statistics did not confirm their results.

\section{The analysis of scanpaths: current approaches}

\subsection{Top-down approaches to the study of visual behavior}

The top-down approach to the study of gaze behaviors concerns the use of areas or regions of interest (AOI or ROI), which are used to describe a Web page. The researcher generally defines these AOIs, and most of the softwares provided by eye-tracking systems manufacturers allow researchers to define, interactively, those regions. These can refer to different visual elements of a Web page such as images and logos, headers and titles, menus, links, paragraphs, search boxes, and so on. But the description of the Web page elements and their grouping can also be done according to their functions (i.e. identification points, reference points).

\subsubsection{Static approaches}

Within the top-down approach, the analyses can be done from static or dynamic point of views. The socalled "static" methods aggregate data such as fixation duration, the number of fixations or the length of scanpaths. Static methods provide a snapshot of the distribution of eye fixations within a Web page for several participants (see [24] for example). Then, it is possible to know which parts of Web pages are attractive or not under various experimental conditions. There are two trends in static methods: top-down and bottom-up approaches.

The top-down approach is characterized by making assumptions on areas viewed by users, sometimes based on certain experimental conditions (ex. type of website, type of task). We call this approach "topdown" because authors assume that some AOI have a meaning or a special role for users, which affects their visual behavior. From a methodological perspective, researchers generate a priori AOIs, which are subject of assumptions. Size or location of the AOIs depends entirely on the research objectives. With this approach, several studies have sought to understand behavioral variability. This is usually done in order to study the effect of different variables on the distribution of various static measures on a Web page or between AOIs. Researchers have thus studied the effects of the type of website [17], the tasks $[4,22]$ or user-related variables such as age [5, $9,10]$, gender $[4,17]$, experience [11] or culture [6] on users' viewing behaviors. 
The bottom-up approach is more marginal in the literature. It consists in observing the overall distribution of eye tracking data on a Web page, without any assumption about their distribution in relation to the web page elements. From the results, authors try to infer a global model of users' eyes behavior. In this category, there is the well-know F-shaped pattern [15], illustrated with a heatmap of the overall fixations distribution of several participants. This pattern is presented as the "dominant reading pattern", and looks like an "F" observed on different websites.

To our knowledge, only one study has attempted to apply this bottom-up approach to study the behavioral variability [21]. The objective was to compare eye patterns of elderly and young people. Results highlight different heatmaps by age group.

Despite their heuristic value, static methods can't reveal the dynamic nature of scanpaths. Behavioral variability is shown only from a static point of view (rate by AOI without order). Thus, no solution is proposed to study the ordered and temporal aspects of scanpaths.

\subsubsection{Dynamic approaches}

Another group of methods is called "dynamic" because they allow us, to some extent, to describe the order and temporal aspects of scanpaths. Thus, scanpaths comparisons can be more precise and detailed, particularly when studying behavioral variability. However, this kind of method is rarely used in the current literature, and is mainly represented by the alignment methods.

Alignment methods involve comparing scanpaths two by two. This comparison is made by transforming the scanpaths into strings of characters (each character corresponding to an AOI). There are two types of alignment methods: the first one is called "local". Its purpose is to find common sub-patterns between scanpaths [23]. The second one is called "global" and allows the comparison of the entire strings. In this second category of alignment methods, the string-edit distance (or Levenshtein distance) consists in comparing the scanpaths pairs by calculating a distance that takes into account the costs of substitution, insertion, and deletion (called indels) needed to transform a string into another [3]. Then, a dissimilarity index of two strings is calculated. Improvements of this string-edit method have been proposed, namely for taking into account common areas between scanpaths [18] and distances between AOIs [20].
Few studies have applied this method on the Web. Josephson \& Holmes [14] were the first to search for the determinants of the scanpaths. Their findings suggest that although subjects have their own viewing habits, other variables such as memory or the characteristics of Web pages seem to play a role on the scanpaths.

Although the string-edit method is the most often used method to study behavioral variability, it has not been developed to compare scanpaths, but to compare strings of characters in words. However, many things distinguish a word from a scanpath, including the notion of temporal order. The Levenshtein distance does not take into account the temporal proximity of viewing the same area between two scanpaths. In addition, there is no method for the definition of an AOI, including its granularity (a region of the Web page, a link, an image, etc.). To overcome AOI limitations, Santella and DeCarlo [19] proposed a method to automatically set AOI from eye fixations (based on Mean-shift clustering). To our knowledge, only Duchowski et al. [8] have applied this clustering method to define AOI and to compare scanpaths.

Recently Jarodzka and Holmqvist [13] tried a new approach based on the comparison of the shape of scanpaths combined with a series of other indicators. The advantage of this method is that it does not require the use of AOI. Nevertheless, the authors have not demonstrated, on real data, its superiority over conventional methods of alignment.

\subsubsection{Limits of the dynamic approaches}

The Levenshtein distance does not take into account the temporal proximity of the same area viewed in two scanpaths. For example, if the subject looks at area $\mathrm{A}$ at time $\mathrm{t}$ while the subject 2 looks at the same area at time $\mathrm{t}+1$, the substitution will cost the same as if the subject 2 looks at this area at time $\mathrm{t}+10$. And if subject 1 has already finished viewing the stimulus to $t+10$, the cost will be that of an indel. Thus, the Levenshtein distance is both insensitive to the order of visualization and very sensitive to the difference in length between scanpaths.

Although the approach of taking into account the AOI is attractive [20], it suffers, however, from an important limitation: the substitution cost depends on the artifact of dividing a priori the screen into AOI. In addition, the temporal order of gaze points is not taken into account. All of this, limits the possibilities of tracking patterns of visual exploration. 


\subsubsection{Finding scan-patterns}

Aula, Majaranta and Räihä [1] attempted to determine the existence of patterns of visualizations on SERPs (Search Engine Result Pages). Their idea was that there are styles of visualization that are more or less effective in achieving a task of information retrieval. To do so, they visually inspected gaze plots of their participants. They then asked independent judges to categorize each participant's gaze plot. Two categories of users were identified.

Hembrooke, Feusner and Gay [12] suggested a method for deriving an "average" scanpath aggregated from many users viewing the same Website. This method uses a multiple sequence alignment algorithm to extract similarities among multiple patterns. Each pattern is then aligned as a sequence with another scan pattern. Using a multiple sequence alignment algorithm to iterate through all the patterns, similarities are preserved in intermediate alignments. The final alignment is a pattern constructed from similarities among the multiple input patterns. Hence this single scan pattern can represent the behavior of the entire group.

In order to characterize and to compare scanpaths so as to uncover possible scan-patterns, Drusch and Bastien [7] has developed a clustering method based on the Hausdorff distance. The results have shown that scan-patterns could be identified: groups of users have been identified and their behaviors have been described with diverse eye-tracking metrics.

The scanpath theory asserts that subjects perform almost the same scan-pattern when they recognize a stimulus. Are these results reproducible on Web pages? According to Josephson and Holmes [14], this would be the case. However, in their experiment, the sample was very small and participants did not have to perform a task. In, addition, the analyses were only based on the Levenshtein distance.

However, many questions remain. For example:

- Are these results reproducible on a large set of participants?

- Are these results confirmed when participants are asked to perform precise tasks on a Web site?

- Are the scanpaths more different between tasks than within task?

- How can we study the variability of scanpaths?

In what follows, we propose a new method for comparing scanpaths and a test of the scanpath theory.

\section{Method}

\subsection{Participants}

On hundred and thirteen French subjects were recruited for the study. Data from 13 subjects were discarded due to calibration problems. Their average age was 23 years old $(\sigma=3.72)$ with an equal proportion of men and women. Most of the subjects $(97,7 \%)$ have used the Internet for at least 3 years and $88.6 \%$ of the participants indicated to be connected on the Web for at least one hour per day.

\subsection{Apparatus}

The Tobii T120 eye-tracking system with a resolution of 1280x1024 Pixels was used for the experiment. The Tobii Studio software (v.1.3.14) was used to manage the test and to extract eye-tracking data. A fixation was defined as a gaze of at least $100 \mathrm{~ms}$ in a radius of 35 pixels.

\subsection{Stimuli}

Two French e-government Websites were selected for this study. Although recordings were performed on all the Web pages participants explored during task completion, only the navigation paths on the homepages are analyzed here.

\subsection{Procedure}

The experiment took place in a laboratory. Subjects were instructed to perform two tasks on each Website without any temporal restrictions (Table 1). All subjects performed the tasks in the same order. First, and between each task, a calibration was performed. All the tasks were presented visually and verbally. Each task started from the homepage of each Website. The use of the keyboard was prohibited so as to prevent eye-tracking data loss, as well as the use of the search engine. 
Table 1

Tasks performed by the participants during the experiment

\begin{tabular}{|l|l|}
\hline \multicolumn{1}{|c|}{ Websites } & \multicolumn{1}{|c|}{ Tasks } \\
\hline service-public.fr & $\begin{array}{l}\text { 1) What are the three cases } \\
\text { allowing to dissolve a civil } \\
\text { pact of solidarity? }\end{array}$ \\
\hline service-public.fr & $\begin{array}{l}\text { 2) What are the first two gen- } \\
\text { eral conditions of recruitment } \\
\text { to access to the public func- } \\
\text { tion? }\end{array}$ \\
\hline carnavalet.paris.fr & $\begin{array}{l}\text { 3) What is the address of the } \\
\text { Municipal Theater of the 15th } \\
\text { district of Paris? }\end{array}$ \\
\hline carnavalet.paris.fr & $\begin{array}{l}\text { 4) What is the price of a full- } \\
\text { conference visit to the Carna- } \\
\text { valet museum? }\end{array}$ \\
\hline
\end{tabular}

\section{Data analysis}

\subsection{Identification of the AOIs}

For the identification of AOIs, we chose to apply Santella and DeCarlo's [19] method witch consist in generating a posteriori AOIs with the help of a clustering method based on the mean-shift algorithm.

AOIs were computed on all scanpaths. A unique identifier was assigned to each AOI. Thus, each scanpath corresponds to a sequence of characters, each character corresponding to a specific AOI. Thus a scanpath corresponds to a sequence of AOIs. Then, these sequences were simplified by removing repetitions [3].

\subsection{Scanpaths comparison: string-edit method revisited}

To meet our objective, we have introduced a new way for calculating the costs of substitution and indels of the string-edit distance so that it best reflects the concept of scanpath (see [2] for more details). In our approach, substitution and insertion take into account the presence or not of a character in two scanpaths (Fig. 1).

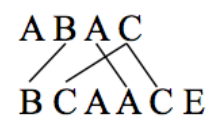

Costs : 120116

Fig. 1. Illustration of our approach: substitution and indels costs are related to the position of characters.

\subsubsection{Substitution costs}

For two scanpaths SP1 and SP2 of length $\mathrm{n}$ and $\mathrm{m}$, the cost "S" of replacing a character $\mathrm{x}$ by another depends on the positions $\mathrm{i}$ and $\mathrm{j}$ of $\mathrm{x}$ respectively in SP1 and SP2. Since many substitutions are possible, only the one that represents the lowest cost must be retained. Thus, we have to find in the second scanpath the occurrence of $x$ closest to i (Eq. 1).

$$
S=|i-j| / \max (n, m)
$$

\subsubsection{Indel costs}

For two scanpaths SP1 and SP2 of length $n$ and m, the indel cost "I" of the character $\mathrm{x}$ depends on the presence or absence of $x$ in both scanpaths. Thus, if the character $\mathrm{x}$ at position $\mathrm{i}$ in SP1 is also present in SP2, the indel cost will depend on the last position $j$ of $x$ in SP2. However, if this character is only present in one of two scanpaths the indel cost will be 1 .

$$
I=(i-j) / \max (n, m)
$$

Using this procedure, a dissimilarity score is generated, for each pair of scanpaths. Thus a dissimilarity matrix may be obtained in which a score is given for all pairs of scanpaths.

\subsection{Common AOIs}

To better analyze the data, another indicator has been introduced. Following Privitera and Stark (2000), the rate of common AOIs between scanpaths (within-subjects) has been computed both for within (repeated tasks) and between tasks.

\subsection{Other indicators}

Various indicators were also computed:

- Temporal indicators: scanpaths total durations (ms), average duration of fixations (ms),

- Average number of fixations,

- Length of scanpaths (pixels),

- Dispersion of scanpaths, weighted by the duration of fixation points. A high dispersion index refers to a dispersed scanpath,

- Convex Hull area (in pixels squared). A large convex Hull area refers to a scanpath with fixation points of high amplitude. 


\section{Results}

To be able to compute within subject statistics, each participant had to provide data for each task and its repetition. Due to technical recording problems only 79 participants out of 100 were included in our analyses.

In the following paragraphs, we present within task (when a participant was asked to repeat the same task) and between tasks results (when a participant was asked to perform a new task on the same web site).

Table 2 presents the dissimilarity scores as well as the rate of common AOIs for between and within tasks.

Table 2.

Dissimilarity scores and rates of common AOIs within and between tasks

\begin{tabular}{|c|c|c|c|}
\hline Type & Tasks & $\begin{array}{l}\text { Dissimilarity } \\
\text { scores }\end{array}$ & $\begin{array}{l}\text { Rate of com- } \\
\text { mon AOIs }\end{array}$ \\
\hline & \multicolumn{3}{|c|}{ Service public } \\
\hline Within task & 1 to $1 \mathrm{R}^{*}$ & 0.71 & 0.93 \\
\hline Between tasks & 1 to 2 & 0.53 & 0.93 \\
\hline \multirow[t]{2}{*}{ Within task } & 2 to $2 \mathrm{R}^{*}$ & 0.79 & 0.48 \\
\hline & \multicolumn{3}{|c|}{ Carnavalet } \\
\hline Within task & 3 to $3 \mathrm{R}^{*}$ & 0.83 & 0.48 \\
\hline Between tasks & 3 to 4 & 0.66 & 0.63 \\
\hline Within task & 4 to $4 \mathrm{R}^{*}$ & 0.71 & 0.60 \\
\hline \multicolumn{2}{|c|}{ Global mean within task } & 0.76 & 0.62 \\
\hline \multicolumn{2}{|c|}{ Global mean between tasks } & 0.59 & 0.78 \\
\hline
\end{tabular}

In general, scanpaths variability (or dissimilarity) between tasks (0.59) appears to be lower than the variability within task (0.76). In other words, when participants repeated the tasks, the scanpaths observed during the first and second time appear to be less similar than the scanpaths observed when performing a different task on the same Web page.

In addition, the rate of common AOIs is lower in the within task condition (0.62) than when achieving a different task $(0.78)$. In other words, when repeating the tasks, participants tended not to look at the same AOIs.

Common AOIs are less frequent within task (2.4 AOIs in average) than between tasks (7.418 AOIs on the average). A student $t$-test for paired samples shows that these differences are statistically significant $(t$ (78) $=-9.945, p=0.0001)$ (Fig. 2).

Scanpaths within tasks seems to be less extended on the Web pages. Indeed, fewer fixations are performed, their lengths are shorter, and scanpaths are less dispersed on the Web page. In addition, fixation durations are longer within tasks (see Table 3).

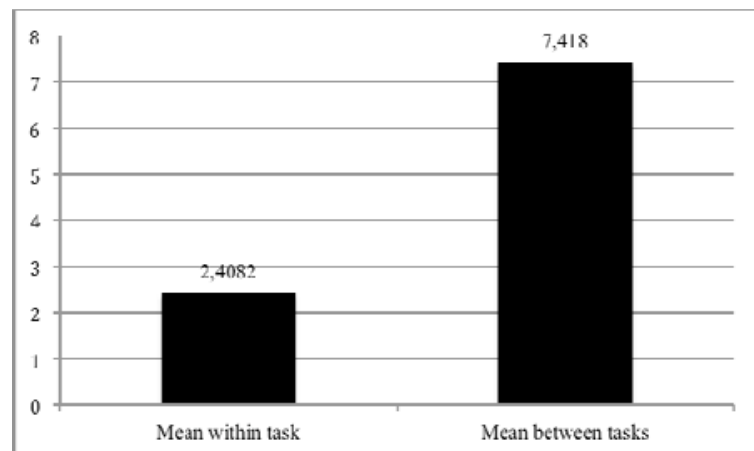

Fig. 2. Mean number of common AOIs within and between tasks.

Table 3

Performances indices for the tasks and their repetitions.

\begin{tabular}{|c|c|c|c|c|c|}
\hline & Mean & $\sigma$ & $t$ & $d f$ & $p$ \\
\hline $\begin{array}{l}\text { Total duration } \\
\text { within task }\end{array}$ & 4423.95 & 2526.67 & \multirow[b]{2}{*}{-16.39} & \multirow[b]{2}{*}{78} & \multirow{2}{*}{.0001} \\
\hline $\begin{array}{l}\text { Total duration } \\
\text { between tasks }\end{array}$ & 18171.60 & 7089.70 & & & \\
\hline $\begin{array}{l}\text { Number of } \\
\text { fixations with- } \\
\text { in tasks }\end{array}$ & 10.14 & 7.27 & \multirow[b]{2}{*}{-14.71} & \multirow[b]{2}{*}{78} & \multirow[b]{2}{*}{.0001} \\
\hline $\begin{array}{l}\text { Number of } \\
\text { fixations be- } \\
\text { tween tasks }\end{array}$ & 44.75 & 19.68 & & & \\
\hline $\begin{array}{l}\text { Fixations dura- } \\
\text { tion within } \\
\text { task }\end{array}$ & 584.90 & 220.71 & \multirow{2}{*}{4.85} & \multirow{2}{*}{78} & \multirow{2}{*}{.0001} \\
\hline $\begin{array}{l}\text { Fixations dura- } \\
\text { tion between } \\
\text { tasks }\end{array}$ & 467.81 & 103.21 & & & \\
\hline $\begin{array}{l}\text { Length within } \\
\text { tasks }\end{array}$ & 1258.48 & 1009.78 & \multirow{2}{*}{-13.54} & \multirow{2}{*}{78} & \multirow{2}{*}{.0001} \\
\hline $\begin{array}{l}\text { Length be- } \\
\text { tween tasks }\end{array}$ & 5981.02 & 2927.54 & & & \\
\hline $\begin{array}{l}\text { Dispersion } \\
\text { within tasks }\end{array}$ & 127.80 & 45.04 & \multirow{2}{*}{-12.19} & \multirow{2}{*}{78} & \multirow{2}{*}{.0001} \\
\hline $\begin{array}{l}\text { Dispersion } \\
\text { between tasks }\end{array}$ & 224.63 & 60.50 & & & \\
\hline $\begin{array}{l}\text { Convex hull } \\
\text { area within } \\
\text { tasks }\end{array}$ & 68337 & 54875 & \multirow{2}{*}{-11.83} & \multirow{2}{*}{78} & \multirow{2}{*}{.0001} \\
\hline $\begin{array}{l}\text { Convex hull } \\
\text { area between } \\
\text { tasks }\end{array}$ & 280550 & 148130 & & & \\
\hline
\end{tabular}

Figure 3 illustrates the analysis of common AOIs within and between tasks for one participant. Between task 1 and task 2, 4 AOIs are common (respectively AOIs 1, 26 and 9). Between task 2 and the repetition of this task (task $2 \mathrm{R}$ ), there is only one common AOI (AOI 8). As we can see in this example, AOI 8 is the last AOI viewed by the participant both on task 2 and the repetition of this task. In addition, the scanpath observed in the repetition of the task is shorter. 
Fig. 3. Illustration of a scanpath in different tasks for a single participant.

\section{Discussion}

In this paper, we have proposed a new method for comparing scanpaths in a bottom-up approach and a test of the scanpath theory. To do so, we conducted a laboratory experiment in which 113 participants were invited to accomplish a set of tasks on two different websites. For each site, they had to perform, in the same order, two tasks that had to be repeated ounce. The data were analyzed using a procedure similar to the one used by Duchowski et al. [8]. The first step was to automatically identify, then label, AOIs with the mean-shift clustering procedure [19]. Then, scanpaths were compared two by two with a modified version of the string-edit method, which take into account the order of AOIs visualizations [2].

Our results show that scanpaths variability between tasks but within participants seems to be lower than the variability within task for a given participant. In other words participants seem to be more coherent when they perform different tasks, than when they repeat the same tasks. In addition, participants view more of the same AOI when they perform a different task on the same Web page than when they repeated the same task.

These results are quite different from what predicts the scanpath theory. If there were a "habitually preferred path" for each participant, the same task, on a Web page, would have caused almost the same viewing behaviors for a given participant.

By studying more deeply the effect of the repetition of a task, we also found that scanpaths tended to be less extensive on the web page than between tasks, as if participants had learnt the organization of the page and wanted to go more directly to the target. This suggests that participants memorize the location that lead to their goal without taking a particular path previously exhibited.

Our results also differ from those of Josephson and Holmes who has observed similar patterns of visual behaviors within participants. Nevertheless, participants in their study had no specific task to perform on their Web pages. Our experimental protocol differs from theirs especially on this point. This suggests that learning occurs when searching for information. People tend to learn the structure of the Web page while searching for information. This may not be the case when peoples' visual exploration behaviors are not guided by an explicit task.

These results should however be interpreted with caution. Indeed, the scanpath theory cannot be invalidated on this basis only. It is in fact possible that repeating many times the same task, would allow a scanpath to appear. Our study does not answer this question. Future work will address this specific aspect.

\section{References}

[1] Aula, A., Majaranta, P., \& Räihä, K.-J. (2005). Eye-Tracking Reveals the Personal Styles for Search Result Evaluation. Human-Computer Interaction-INTERACT 2005, 1058-1061.

[2] Bastien, J. M. C., \& Drusch, G. (2011). Exploring web pages... Are all the people really exhibit a F-shaped pattern? Paper presented at the $11^{\circ}$ Congresso Internacional de Ergonomia e Usabilidade de Interfaces Humano-Comutador, Manaus, Brazil.

[3] Brandt, S., \& Stark, L. (1997). Spontaneous eye-movements during visual imagery reflect the content of the visual scene. Journal of Cognitive Neuroscience, 9(1), 27-38.

[4] Buscher, G., Cutrell, E., \& Morris, M. R. (2009). What do you see when you're surfing?: using eye tracking to predict salient regions of web pages. Paper presented at the Proceedings of the 27 th international conference on Human factors in computing systems.

[5] Djamasbi, S., Siegel, M., \& Tullis, T. (2010). Generation Y, web design, and eye tracking. International Journal of Human-Computer Studies, 68, 307-323.

[6] Dong, Y., \& Lee, K.-P. (2008). A Cross-Cultural Comparative Study of Users 'Perceptions of a Webpage : With a Focus on the Cognitive Styles of Chinese , Koreans and Americans Eye Tracking in Usability Testing. International Journal of Design, 2, 19-30.

[7] Drusch, G., Bastien, J. M. C., \& Dinet, J. (2011). From gaze plots to eye fixation patterns using a clustering method based on Hausdorff distances. Paper presented at the IHM'2011, Nice, France.

[8] Duchowski, A. T., Driver, J., Jolaoso, S., Tan, W., Ramey, B. N., \& Robbins, A. (2010). Scanpath comparison revisited. Paper presented at the Proceedings of the 2010 Symposium on Eye-Tracking Research \& Applications - ETRA '10, New York, New York, USA.

[9] Fukuda, R., \& Bubb, H. (2003). Eye tracking study on Webuse: Comparison between younger and elderly users in case 
of search task with electronic timetable service. PsychNology Journal, 1, 202-228.

[10] Grahame, M., Laberge, J., \& Scialfa, C. (2004). Age differences in search of Web page : the effects of links size, link number and clutter. Human factors, 46, 385-398.

[11] Habuchi, Y., Takeuchi, H., \& Kitajima, M. (2006). The influence of web browsing experience on web-viewing behavior. Paper presented at the Proceedings of the 2006 symposium on Eye tracking research \& applications - ETRA '06, New York, New York, USA.

[12] Hembrooke, H., Feusner, M., \& Gay, G. (2006). Averaging scan patterns and what they can tell us. Paper presented at the Proceedings of the 2006 symposium on Eye tracking research \& applications - ETRA '06, New York, New York, USA.

[13] Jarodzka, H., Holmqvist, K., \& Nyström, M. (2010). A vector-based, multidimensional scanpath similarity measure. Paper presented at the Proceedings of the 2010 Symposium on Eye-Tracking Research and Applications, Austin, Texas.

[14] Josephson, S., \& Holmes, M. E. (2002,). Attention to repeated images on the World-Wide Web: Another look at scanpath theory. Behavior Research Methods, Instruments, \& Computers, 34(4), 539-548.

[15] Nielsen, J. (Producer). (2006). F-Shaped Pattern For Reading Web Content. Jakob Nielsen's Alertbox. Retrieved from $\mathrm{http}: / /$ www.useit.com/alertbox/reading pattern.html

[16] Noton, D., \& Stark, L. (1971). Scanpaths in Eye Movements during Pattern Perception. Science, 171, 308-311.

[17] Pan, B., Hembrooke, H. A., Gay, G. K., Granka, L. A., Feusner, M. K., \& Newman, J. K. (2004). The determinants of web page viewing behavior: an eye-tracking study. Paper presented at the Proceedings of the 2004 symposium on Eye tracking research \& applications.
[18] Privitera, C. M., \& Stark, L. W. (2000). Algorithms for Defining Visual Regions-of-Interest: Comparison with Eye Fixations. IEEE Trans. Pattern Anal. Mach. Intell., 22(9), 970-982. doi: $10.1109 / 34.877520$

[19] Santella, A., \& DeCarlo, D. (2004). Robust Clustering of Eye Movement Recordings for Quantification of Visual Interest. Paper presented at the ETRA 2004

[20] Takeuchi, H., \& Habuchi, Y. (2007). A quantitative method for analysing scanpath data obtained bu eye tracker. Paper presented at the IEEE Symposium on computational intelligence and data mining (CIDM 2007).

[21] Tullis, T. S. (2007). Older adults and the web: lessons learned from eye-tracking. Paper presented at the Proceedings of the 4th international conference on Universal access in human computer interaction: coping with diversity, Beijing, China.

[22] Tzanidou, E., Petre, M., Minocha, S., \& Grayson, A. (2005). Combining Eye Tracking and Conventional Techniques for Indications of User-Adaptability. Paper presented at the INTERACT 2005, New York, USA.

[23] West, J. M., Haake, A. R., Rozanski, E. P., \& Karn, K. (2006, 27-29 March 2006). eyePatterns: Software for Identifying Patterns and Similarities Across Fixation Sequences. Paper presented at the ETRA 2006, San Diego, California, USA.

[24] Yesilada, Y., Jay, C., Stevens, R., \& Harper, S. (2008). Validating the use and role of visual elements of web pages in navigation with an eye-tracking study. In Proceeding of the 17th international conference on World Wide Web (pp. 11-20). New York, USA: ACM. 